\title{
A PDZ-interacting domain in CFTR is an apical membrane polarization signal
}

\author{
Bryan D. Moyer, ${ }^{1}$ Jerod Denton, ${ }^{1}$ Katherine H. Karlson, ${ }^{1}$ Donna Reynolds, ${ }^{1}$ \\ Shusheng Wang, ${ }^{2}$ John E. Mickle, ${ }^{3}$ Michal Milewski, ${ }^{3}$ Garry R. Cutting, ${ }^{3}$ \\ William B. Guggino, ${ }^{4}$ Min Li, ${ }^{2}$ and Bruce A. Stanton ${ }^{1}$
}

\begin{abstract}
${ }^{1}$ Department of Physiology, Dartmouth Medical School, Hanover, New Hampshire 03755, USA
${ }^{2}$ Departments of Physiology and Neuroscience, Johns Hopkins University School of Medicine, Baltimore, Maryland 21205, USA

${ }^{3}$ Center for Medical Genetics, Johns Hopkins Hospital, Baltimore, Maryland 21287, USA

${ }^{4}$ Department of Physiology, Johns Hopkins University School of Medicine, Baltimore, Maryland 21205, USA
\end{abstract}

Address correspondence to: Bruce A. Stanton, Department of Physiology, Dartmouth Medical School, Hanover, New Hampshire 03755, USA. Phone: (603) 650-1775; Fax: (603) 650-1130; E-mail: Bruce.A.Stanton@Dartmouth.edu.

Received for publication May 28, 1999, and accepted in revised form October 5, 1999.

\begin{abstract}
Polarization of the cystic fibrosis transmembrane conductance regulator (CFTR), a cAMP-activated chloride channel, to the apical plasma membrane of epithelial cells is critical for vectorial transport of chloride in a variety of epithelia, including the airway, pancreas, intestine, and kidney. However, the motifs that localize CFTR to the apical membrane are unknown. We report that the last 3 amino acids in the $\mathrm{COOH}$-terminus of CFTR (T-R-L) comprise a PDZ-interacting domain that is required for the polarization of CFTR to the apical plasma membrane in human airway and kidney epithelial cells. In addition, the CFTR mutant, S1455X, which lacks the $26 \mathrm{COOH}$-terminal amino acids, including the PDZ-interacting domain, is mispolarized to the lateral membrane. We also demonstrate that CFTR binds to ezrin-radixin-moesin-binding phosphoprotein 50 (EBP50), an apical membrane PDZ domain-containing protein. We propose that $\mathrm{COOH}$-terminal deletions of CFTR, which represent about $10 \%$ of CFTR mutations, result in defective vectorial chloride transport, partly by altering the polarized distribution of CFTR in epithelial cells. Moreover, our data demonstrate that PDZ-interacting domains and PDZ domain-containing proteins play a key role in the apical polarization of ion channels in epithelial cells.
\end{abstract}

J. Clin. Invest. 104:1353-1361 (1999).

\section{Introduction}

The cystic fibrosis transmembrane conductance regulator (CFTR) is a member of the ATP-binding cassette family and functions as a cAMP-activated chloride channel that is polarized to the apical plasma membrane in epithelial cells (1-3). Mutations in the CFTR gene lead to the genetic disease cystic fibrosis (CF), a lethal autosomal recessive disorder affecting approximately 1 in 2,000 live births in Caucasians (4-6). CF is characterized by high sweat $\mathrm{Cl}^{-}$concentration, pulmonary disease, and pancreatic insufficiency, although many other organs including the kidney, liver, heart, and organs of the reproductive tract are also affected $(4,5)$. More than 800 different mutations have been identified within the CFTR gene (7). Nearly $70 \%$ of individuals with CF are homozygous for the CFTR- $\Delta$ F508 mutation, which accounts for approximately $90 \%$ of all mutant CFTR alleles (5). CFTR- $\triangle$ F508 is a folding mutation that is trapped in the endoplasmic reticulum and does not traffic to the apical plasma membrane $(8,9)$. However, CFTR- $\Delta$ F508 retains function as a cAMP-activated $\mathrm{Cl}^{-}$ channel (10). Therefore, identification of strategies for increasing anterograde trafficking of CFTR- $\Delta$ F508 through the secretory pathway to the apical plasma membrane, where it can function as a cAMP-activated $\mathrm{Cl}^{-}$channel, would have important therapeutic implications for the treatment of CF. To devise strategies for stimulating CFTR- $\Delta$ F508 trafficking to the apical plasma membrane, it is first necessary to identify the sorting determinants that direct CFTR to the apical membrane in polarized epithelial cells. However, nothing is known about the motifs involved in directing the polarized expression of CFTR in the apical plasma membrane.

The generation and maintenance of cell polarity are crucial for vectorial solute and fluid transport in epithelial cells and for the normal function of neurons (11-15). Epithelial cells must asymmetrically distribute receptors, transporters, ion channels, and lipids between the apical and basolateral membranes to establish and maintain polarity and function $(11,13,14,16)$. Neurons also must asymmetrically distribute proteins between axons and somatodendritic regions (12). In general, proteins sorted to the apical membrane of epithelia are expressed in the axons of neurons, whereas proteins sorted to the basolateral membrane of epithelial are distributed to the somatodendritic region of neurons (12). Polarization of proteins to apical (axon) and basolateral membrane (somatodendritic) domains is achieved by distinct sorting or retention signals. Basolateral membrane sorting signals reside in the cytoplasmic domains of transmembrane proteins and are composed of short peptide motifs including tyrosine-dependent signals (i.e., NPXY or YXX $\varnothing$, where $X$ is any amino acid and $\varnothing$ is a large hydrophobic amino acid such as leucine, isoleucine, or 
valine), tyrosine-independent signals, and dileucinebased motifs $(13,14)$. Some integral membrane proteins such as $\mathrm{Na}^{+}-\mathrm{K}^{+}$-ATPase $(17,18)$ and $\beta 1$-integrin $(19)$ are not sorted preferentially to either the apical or basolateral membrane from the trans-Golgi network but are selectively retained and accumulated in the basolateral membrane by interaction with the membrane-associated cytoskeleton. By contrast, less is known about the signals that sort and/or retain proteins in the apical (axon) plasma membrane. Apical membrane sorting signals frequently reside in transmembrane or extracellular domains and are often composed of lipid or polysaccharide groups. Three classes of apical membrane sorting signals have been identified: glycosylphosphatidylinositol (GPI) membrane anchors, $N$ - and $O$-linked polysaccharides, and transmembrane domains $(13,14)$.

Recent studies have demonstrated that PDZ domains, which are named for 3 proteins in which this domain was first described (PSD-95, Dlg, and ZO-1), play an essential role in determining cell polarity (20). PDZ domains are modular 70-90 amino acid domains in proteins that bind to short peptide sequences at the $\mathrm{COOH}$-termini of other proteins, called PDZ-interacting domains (20-23). PDZ domain-PDZ-interacting domain interaction localizes some proteins to the basolateral plasma membrane $(20,24-27)$. A role for PDZ domains in the localization of proteins to the apical membrane is also emerging $(28,29)$. Recently, we and others reported that the $\mathrm{COOH}$-terminal amino acids of CFTR (T-R-L) are highly conserved and comprise a PDZ-interacting domain that binds to PDZ domains in ezrin-radixin-moesin-binding phosphoprotein 50 (EBP50), a protein that is polarized to the apical membrane in MDCK and other epithelial cells (30-32). Because recent studies implicate PDZ domains in the polarization of proteins to the apical membrane (28, 29 ), and because nothing is known about the signals that determine the apical polarization of CFTR, the objective of the present study was to test the hypothesis that the PDZ-interacting domain (T-R-L) in CFTR functions as an apical membrane polarization signal. Toward these ends, we made chimeric constructs in which the green fluorescent protein (GFP) was linked to either wild-type (wt) CFTR or CFTR with truncations in the COOH-terminus (i.e., CFTR- $\Delta$ TRL or CFTR$\mathrm{S} 1455 \mathrm{X}$ ) and expressed these proteins in polarized kidney epithelial cells (MDCK) and human bronchial epithelial cells (16HBE14o-). We report that the PDZinteracting domain of CFTR is required for the polarization of CFTR to the apical plasma membrane. In addition, the CFTR mutation S1455X, which lacks the $26 \mathrm{COOH}$-terminal amino acids including the PDZinteracting domain, is mispolarized to the lateral membrane. We also demonstrate that CFTR binds to EBP50, an apical membrane PDZ domain-containing protein. We propose that $\mathrm{COOH}$-terminal deletions of CFTR result in defective vectorial chloride transport in epithelial cells in part by altering the polarized distribution of CFTR. In addition, our data demonstrate that PDZinteracting domains and PDZ domain-containing proteins play a key role in the apical polarization of ion channels in epithelial cells.

\section{Methods}

Fusion constructs. pGFP-CFTR encoding enhanced GFP fused to the $\mathrm{NH}_{2}$-terminus of wt-CFTR, was constructed as described (33). Previously, we demonstrated that addition of GFP to the $\mathrm{NH}_{2}$-terminus of CFTR had no effect on CFTR localization, trafficking, or function (33). Moreover, others have shown that GFP has no effect on the biosynthesis or degradation of CFTR (34). As described later here, $\mathrm{COOH}$-terminal deletion constructs of CFTR were generated by PCR-based mutagenesis. PCR primers were designed using Oligo v4.04 Primer Analysis Software (Plymouth, Minnesota, USA) and synthesized by the Dartmouth College Molecular Biology Core Facility (Hanover, New Hampshire, USA). Automated DNA sequence analysis of PCR products and deletion constructs was performed using the ABI PRISM Dye Terminator Cycle Sequencing Ready Reaction Kit (Perkin Elmer Corp., Norwalk, Connecticut, USA) to confirm the desired deletion and to verify that base misincorporation did not occur during PCR. pGFP-CFTR- $\triangle$ TRL encodes a GFP-CFTR fusion protein lacking the $3 \mathrm{COOH}$-terminal amino acids. Oligonucleotide primers (sense $5^{\prime}$ TAA ACC TAC CAA GTC AAC CAA ACC ATA C 3' and antisense 5' CGA TAT CAT CTA ATC TTG CAT CTC TTC TTC TGT CTC CTC $\left.3^{\prime}\right)$ were used to amplify a 923-bp fragment from pGFPCFTR plasmid DNA (underlined sequences in the antisense primer correspond to an artificial EcoRV site appended to facilitate cloning; bold sequences correspond to the introduced CFTR premature stop codon). The PCR product was electrophoretically separated on $1 \%$ low melting point agarose gel (Life Technologies, Gaithersburg, Maryland, USA), gel-purified using the Wizard PCR Preps DNA Purification System (Promega Corp., Madison, Wisconsin, USA), and subcloned into the PCR 2.1 vector using the TA Cloning Kit (Invitrogen, San Diego, California, USA). After sequence analysis, the PCR product was excised from pCR 2.1 using BstX1 and EcoRV and subcloned into pGFP-CFTR. pGFP-CFTR-S1455X encodes a GFP-CFTR fusion protein lacking the $26 \mathrm{CFTR} \mathrm{COOH}-$ terminal amino acids due to mutation of codon 1455 (which encodes serine) to a premature stop codon. Oligonucleotide primers (sense 5' GCC AAC AGA AGG TAA ACC TAC CAA GTC AAC CAA ACC ATA C $3^{\prime}$ and antisense 5' CGA TAT C TCA GTT CCG GTG GGG AAA GAG C $3^{\prime}$ ) were used to amplify an 866-bp fragment from pGFP-CFTR (underlined sequences in the antisense primer correspond to an artificial EcoRV site appended to facilitate cloning; bold sequences correspond to the introduced CFTR premature stop codon). After sequence analysis, the PCR product was excised from PCR 2.1 using BstX1 and EcoRV and subcloned into pGFP-CFTR. pHAEBP50 encodes mouse EBP50 tagged with the hemagglutinin (HA) epitope at the $\mathrm{NH}_{2}$-terminus and was constructed as described elsewhere (32).

Cell culture and transfection. MDCK type I cells stably expressing GFP-CFTR fusion proteins were grown at $33^{\circ} \mathrm{C}$ on Transwell permeable filter supports (Corning Inc., Corning, New York, USA) as described previously (33). COS-7 cells were obtained from the American Type Tissue Collection (CRL-1651; Rockville, Maryland, USA) and grown on tissue culture-treated polystyrene flasks in DMEM (JRH Biosciences, Lenexa, Kansas, USA) con- 
taining $0.37 \%$ sodium bicarbonate, $10 \%$ FBS (Hyclone, Logan, UT), $50 \mathrm{U} / \mathrm{mL}$ penicillin, $50 \mathrm{mg} / \mathrm{mL}$ streptomycin, and $2 \mathrm{mM}$ L-glutamine (Life Technologies) in 5\% $\mathrm{CO}_{2}$-balanced air at $37^{\circ} \mathrm{C}$. Human airway epithelial cells (16HBE14o-), expressing low levels of endogenous wtCFTR, a generous gift of D. Gruenert (University of California at San Francisco, San Francisco, California, USA), were grown on VPM-coated Millicell PCF permeable filter supports (Millipore Corp., Bedford, Massachusetts, USA) in MEM with Earle's salts containing 10\% FBS, 50 $\mathrm{U} / \mathrm{mL}$ penicillin, $50 \mathrm{mg} / \mathrm{mL}$ streptomycin, and $2 \mathrm{mM}$ Lglutamine. COS-7 cells and 16HBE14o- cells were transiently transfected using lipofectamine (Life Technologies) following the manufacturer's instructions.

Immunofluorescence microscopy. Cells were fixed and stained for the tight junction protein $\mathrm{ZO}-1$, the lateral membrane marker protein $\mathrm{Na}^{+}-\mathrm{K}^{+}$-ATPase, or biotinylated surface membrane proteins as described previously (33). HA-tagged EBP50 was immunolocalized by indirect immunofluorescence using anti-HA mAb 12CA5. Cells were fixed in $3 \%$ paraformaldehyde for 20 minutes at room temperature, washed in PBS, and permeabilized with $0.2 \% \mathrm{NP}-40$ for 15 minutes. After washing in PBS, nonspecific binding sites were blocked with PBS/10\% normal goat serum (NGS; DAKO Corp., Carpinteria, California, USA) for 30 minutes. Cells were stained with 1:500 anti-HA mAb in PBS/5\% NGS, washed with PBS/1\% BSA, and incubated with 1:100 goat anti-mouse Texas Red secondary antibody (Molecular Probes Inc., Eugene, Oregon, USA) in PBS/1\% NGS. Cells were washed and mounted in $90 \%$ glycerol $/ 10 \%$ PBS containing $10 \mathrm{mg} / \mathrm{mL} n$-propyl gallate to retard fading. Cells were imaged by confocal microscopy as described (33).

Selective cell-surface biotinylation and coimmunoprecipitation. Selective apical and basolateral membrane biotinylations were performed on at least 3 separate clones for wt and mutant CFTR as described (33). All steps were performed at $4^{\circ} \mathrm{C}$. For coimmunoprecipitation, COS-7 cells were solubilized in lysis buffer $(50 \mathrm{mM}$ Tris $\mathrm{HCl}[\mathrm{pH}$ 8.0], $150 \mathrm{mM} \mathrm{NaCl}$, and $1 \%$ Triton X-100, containing the Complete Protease Inhibitor cocktail [Boehringer Mannheim, Indianapolis, Indiana, USA]) for 15-20 minutes. Cells were disrupted mechanically, and lysates were spun at $14,000 \mathrm{~g}$ for 4 minutes at $4^{\circ} \mathrm{C}$ to pellet insoluble material. One tenth of cell supernatant volumes, defined as total cell lysates, were removed for analysis of total cell GFP-CFTR and HA-EBP50 content by SDS-PAGE. Remaining supernatants were precleared with a $50 \%$ slurry of Protein A-agarose beads (Pierce Chemical Co., Rockford, Illinois, USA) for $1-2$ hours at $4^{\circ} \mathrm{C}$ with endover-end rotation. Beads were pelleted by brief centrifugation (15 seconds at 14,000 g). GFP-CFTR fusion proteins were immunoprecipitated with $5 \mu \mathrm{g}$ polyclonal GFP antibody (CLONTECH Laboratories Inc., Palo Alto, California, USA) or $5 \mu \mathrm{g}$ nonimmune IgG control rabbit antibody (DAKO) for 3-4 hours at $4^{\circ} \mathrm{C}$ with end-overend rotation. Antibody-antigen complexes were precipitated with Protein A-agarose beads and incubated for $2-3$ hours at $4{ }^{\circ} \mathrm{C}$ with end-over-end rotation. Beads were pelleted by brief centrifugation as already described here, washed (3 times) with lysis buffer, and rotated end-overend for 5 minutes at $4^{\circ} \mathrm{C}$. Immunoprecipitated proteins were eluted from beads with SDS sample buffer $(0.24 \mathrm{M}$ Tris $\mathrm{HCl}$ [pH 8.9], $16 \%$ glycerol, $0.008 \%$ bromophenol blue, $5.6 \%$ SDS, and $80 \mathrm{mM}$ of freshly prepared DTT) at $95^{\circ} \mathrm{C}$ for 5 minutes.

SDS-PAGE and Western blotting. Proteins were separated by SDS-PAGE and electrophoretically transferred to PVDF membranes as described elsewhere (33). GFPCFTR fusion proteins were detected on membranes blocked with $5 \%$ nonfat dry milk in TBS/0.02\% Tween20 using monoclonal GFP antibody (1:1,000; CLONTECH) or monoclonal CFTR COOH-terminal antibody (1:1,000; Genzyme Pharmaceuticals, Cambridge, Massachusetts, USA) followed by anti-mouse HRP $(1: 5,000$; Amersham Life Sciences Inc., Arlington Heights, Illinois, USA). HA-EBP50 was detected on membranes blocked with Superblock (Pierce Chemical Co.) supplemented with $0.02 \%$ Tween-20 using biotinylated anti-HA mAb 12CA5 (1:200; Boehringer-Mannheim) followed by neutravidin-HRP (1:80,000; Pierce Chemical Co.). Both HA antibody and neutravidin-HRP were prepared in Superblock containing $0.02 \%$ Tween- 20 .

In MDCK cells stably expressing GFP-CFTR- $\Delta$ TRL and GFP-wt-CFTR, but not in untransfected parental cells, the GFP mAb recognized 2 bands with relative molecular masses of approximately $210-240 \mathrm{kDa}$. These bands represent core glycosylated (210-kDa band B) and mature glycosylated (240-kDa band C) GFP-CFTR as described previously (33). In cells expressing GFP-CFTR-S1455X, the GFP mAb recognized 2 bands with relative molecular masses of approximately 210 and $240 \mathrm{kDa}$. As expected, an antibody that recognizes an epitope corresponding to the last 4 amino acids of CFTR $(35,36)$ only labeled GFP-wtCFTR and did not detect GFP-CFTR- $\triangle$ TRL or GFP-CFTR1455X. These observations confirm that the GFP-CFTR$\triangle T R L$ and GFP-CFTR-1455X cDNAs encode truncated CFTR proteins lacking COOH-terminal amino acids.

Semiquantitative confocal microscopy. In 16HBE14o- cells, GFP-CFTR expression was too low to perform domain selective cell-surface biotinylation to quantitate apical versus basolateral membrane distribution. Thus, we examined the relative distribution of GFP-CFTR fluorescence in the apical and lateral membranes by semiquantitative confocal microscopy. GFP fluorescence was quantitated in randomly acquired confocal micrograph xz vertical sections as described previously, with minor modifications (37). Using NIH Image software (version 1.57; National Institutes of Health, Bethesda, Maryland, USA), a box ( 2$\mu \mathrm{m}$ wide and encompassing the length of apical or lateral membranes) was drawn over the region to be measured, and pixel counts within the boxed region were determined. The transition between apical and lateral membranes was identified by staining monolayers for the tight junction protein $\mathrm{ZO}-1$. Pixel intensity histograms were evaluated to ensure that GFP fluorescence was within the linear range (0-254). In stable transfectants, apical to basolateral polarity ratios $(R)$ were calculated using the following formula: $R=(\mathrm{a}-\mathrm{c}) /\left[\left(\mathrm{b}_{\mathrm{l}}-\mathrm{c}\right) / 2+\left(\mathrm{b}_{\mathrm{r}}-\mathrm{c}\right) / 2\right]$ where $a$ corresponds to pixel counts in the apical membrane region, $b_{l}$ corresponds to pixel counts in the left lateral membrane region, $b_{r}$ corresponds to pixel counts in the right lateral membrane region, and $c$ corresponds to background pixel counts in the nucleus. Lateral membrane 


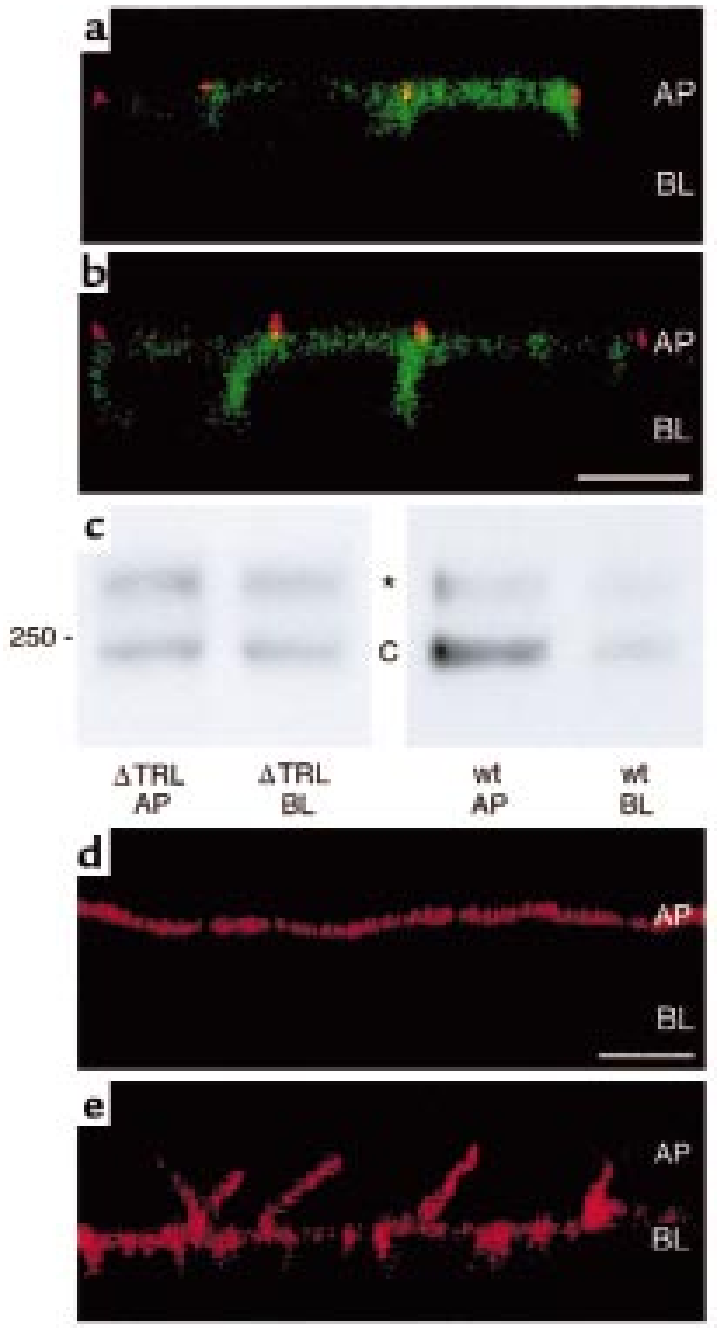

\section{Figure 1}

The $\mathrm{COOH}$-terminal PDZ-interacting domain (TRL) is required for polarization of CFTR to the apical membrane. (a) Confocal fluorescence micrograph (xz plane) of MDCK cells stably expressing GFP-wtCFTR in the apical membrane. (b) Confocal fluorescence micrograph (xz plane) of MDCK cells stably expressing GFP-CFTR- $\Delta T R L$ in the api$\mathrm{cal}$ and lateral membranes. GFP fluorescence is green, and ZO-1, a protein in tight junctions that separates apical and basolateral membrane domains, is red. Scale bar $=10 \mu \mathrm{m}$. AP = location of apical membrane; $B L=$ location of basal membrane. (c) Western blot of cells expressing wt-CFTR or CFTR- $\Delta T R L$. Selective cell-surface biotinylation of the apical (AP) or basolateral membrane (BL). Whereas GFP-wt-CFTR was expressed primarily in the apical membrane (ratio of apical/basolateral membrane expression $=7.5$ ), GFP-CFTR- $\Delta T R L$ was expressed nearly equally in the apical and basolateral membranes (ratio of apical/basolateral membrane expression $=0.6$ ). Mature, glycosylated GFP-wt-CFTR band C is approximately $240 \mathrm{kDa} .{ }^{*} \mathrm{High}$ molecular weight form of CFTR that has been reported previously (33, 37). (d) Selective biotinylation of the apical membrane. (e) Selective biotinylation of the basolateral membrane. Biotin was detected with streptavidin-Texas-red as described (33). Images in $\mathbf{d}$ and $\mathbf{e}$ demonstrate that in our experiments, tight junctions were intact and the biotin regent applied to the apical membrane did not have access to CFTR present on the basolateral membrane and vice versa. In addition, the absence of core-glycosylated GFP-wt-CFTR (band B) in surface biotinylated samples indicates that cell integrity was not compromised and that biotin was not accessible to the endoplasmic reticulum and cis-Golgi apparatus, where core glycosylated CFTR band $B$ is located. measurements were divided by 2 when adjacent cells, with opposing lateral surfaces, displayed GFP fluorescence. The denominator was not divided by 2 in measurements of transiently transfected cells, because only single GFPpositive cells were observed. GFP fluorescence was generally not observed in the basal membrane that was excluded from analyses. A similar method has previously been used to quantitate the apical to basolateral polarity ratios of $\mathrm{Na}^{+}-\mathrm{K}^{+}$-ATPase and $\mathrm{H}^{+}-\mathrm{K}^{+}$-ATPase subunits (37). In stably transfected MDCK cells, we obtained similar results using domain selective cell-surface biotinylation and semiquantitative confocal microscopy to determine the ratio of apical to basolateral expression of CFTR (Table 1).

\section{Results}

To determine whether the COOH-terminal PDZ-interacting domain (T-R-L) is important for the polarization of CFTR to the apical membrane of epithelial cells, we stably expressed GFP-wt-CFTR, GFP-CFTR- $\Delta$ TRL, or GFP-CFTR-1455X in MDCK cells. Examination of GFPwt-CFTR localization by immunofluorescence confocal microscopy demonstrated that GFP-wt-CFTR was polarized to the apical plasma membrane (Figure 1a). The ratio of GFP-wt-CFTR in the apical versus the basolateral plasma membrane was $7.5 \pm 2.3$ as determined by domainselective cell-surface biotinylation (Figure 1c; Table 1). By contrast, examination of GFP-CFTR- $\Delta$ TRL localization by immunofluorescence confocal microscopy demonstrated that GFP-CFTR- $\Delta$ TRL was not polarized to either the apical or basolateral plasma membranes (Figure $1 \mathrm{~b}$ ). The ratio of GFP-CFTR- $\Delta$ TRL in the apical versus the basolateral membrane was $0.6 \pm 0.3(P<0.001$ versus wtCFTR; Table 1) as determined by domain-selective cellsurface biotinylation (Figure 1c). Similar results were obtained in human airway epithelial cells (16HBE14o-; Table 1). Examination of GFP-wt-CFTR distribution in 16HBE14o- cells by immunofluorescence confocal microscopy demonstrated that wt-CFTR was polarized to the apical plasma membrane. By contrast, GFP-CFTR$\triangle$ TRL was not polarized to either the apical or basolateral plasma membrane in $16 \mathrm{HBE} 14 \mathrm{o}-$ cells. The ratio of GFP-wt-CFTR in the apical versus the basolateral membrane of $16 \mathrm{HBE} 14 \mathrm{o}$ - cells was $3.6 \pm 0.6$, whereas the ratio of GFP-CFTR- $\triangle$ TRL in the apical versus the basolateral membrane was $0.7 \pm 0.1$ ( $P<0.001$ versus wt-CFTR; Table 1). Thus, the PDZ-interacting domain (T-R-L) is required for the apical polarization of CFTR in kidney (MDCK) and human airway epithelial cells (16HBE14o-).

Approximately $10 \%$ of all mutations in CFTR result in the deletion of the $\mathrm{COOH}$-terminal region including the PDZ-interacting domain. Because deletion of 61 or fewer amino acids in the $\mathrm{COOH}$-terminus of CFTR does not alter cAMP-stimulated $\mathrm{Cl}^{-}$permeability $(38,39)$, the mechanism whereby these mutations cause CF is unclear. Accordingly, we examined the cellular distribution of the COOH-terminal truncation mutant, S1455X, which lacks the $\mathrm{COOH}$-terminal 26 amino acids including the PDZ-interacting domain (40). Patients with CFTRS1455X have defective $\mathrm{Cl}^{-}$transport in sweat ducts (40). Examination of GFP-CFTR-S1455X localization by immunofluorescence confocal microscopy demonstrated that CFTR-S1455X was polarized to the lateral plasma 


\section{Table 1}

Deletion of the PDZ-interacting domain of CFTR abrogates its apical polarization

\begin{tabular}{lccc}
\hline Cell line & wt-CFTR & CFTR- $\Delta$ TRL & CFTR-S1455X \\
MDCK (Biotinylation) & $7.5 \pm 2.3$ & $0.6^{\mathrm{A}} \pm 0.3$ & $0.2^{\mathrm{A}} \pm 0.1$ \\
MDCK (Confocal) & $3.0 \pm 0.4$ & $0.6^{\mathrm{A}} \pm 0.1$ & $0.1^{\mathrm{A}} \pm 0.1$ \\
16HBE14o-(Confocal) & $3.6 \pm 0.6$ & $0.7^{\mathrm{A}} \pm 0.1$ & $0.1^{\mathrm{A}} \pm 0.1$
\end{tabular}

Data are expressed as the ratio of CFTR expressed in the apical versus the basolateral plasma membrane. The polarized expression of CFTR was determined by selective, cell-surface biotinylation or by semiquantitative, confocal microscopy as described in Methods. Similar results were obtained in MDCK cells using both methods, validating the semiquantitative confocal microscopy approach. Data are expressed as mean \pm SEM. ASignificantly different from wt-CFTR in the same cell line $(P<0.001)$.

membrane of kidney (MDCK) and human airway epithelial cells (16HBE14o-; Figure 2 and Table 1). GFP-CFTR$\mathrm{S} 1455 \mathrm{X}$ colocalized with the lateral membrane protein $\mathrm{Na}^{+}-\mathrm{K}^{+}$-ATPase (Figure 2d). Moreover, the ratio of GFPCFTR-S1455X in the apical versus the basolateral plasma membrane was $0.2 \pm 0.1$ as determined by domain-selective cell-surface biotinylation (Table 1). These results confirm that the $\mathrm{COOH}$-terminus of CFTR is required for the apical polarization of CFTR in kidney (MDCK) and human airway epithelial cells (16HBE14o-). That CFTRS1455X was expressed primarily in the basolateral membrane, whereas CFTR- $\Delta$ TRL was equally distributed in the apical and basolateral plasma membranes, suggests that some basolateral targeting information is suppressed or inactive in CFTR- $\Delta$ TRL (see Discussion).

We and others recently reported that the PDZ domain-containing protein EBP50, which is expressed in MDCK and human airway epithelial cells, binds to the PDZ-interacting domain of CFTR as determined by yeast 2 -hybrid analysis and in vitro pulldown assays (30-32). However, interaction and colocalization of CFTR and EBP50 in vivo have not been reported. To determine whether EPB50 interacts with and colocalizes with GFPwt-CFTR in vivo, and whether the PDZ-interacting domain of CFTR is required for interaction and colocalization with EBP50, we coexpressed EBP50 with GFP-wtCFTR, GFP-CFTR- $\triangle T R L$, or GFP-CFTR-S1455X in MDCK and COS cells. Examination of GFP-wt-CFTR and EBP50 by immunofluorescence confocal microscopy demonstrated that GFP-wt-CFTR and EBP50 colocalized to the apical plasma membrane of MDCK cells (Figure 3, a-c). However, GFP-CFTR- $\Delta$ TRL, which was expressed equally in the apical and lateral membranes, only partially colocalized with EBP50 that was expressed in the apical plasma membrane. Colocalization of GFP-CFTR$\triangle T R L$ was evident in the apical but not in the lateral membranes (Figure 3, d-f). By contrast, examination of GFP-CFTR-S1455X location by immunofluorescence confocal microscopy demonstrated that GFP-CFTRS1455X did not colocalize with EBP50. GFP-CFTRS1455X was polarized to the lateral plasma membrane, whereas EBP50 was polarized to the apical plasma membrane (Figure 3, g-i).

To test the hypothesis that EBP50 and CFTR interact via the $\mathrm{COOH}$-terminal $\mathrm{PDZ}$-interacting domain of CFTR in vivo, we coexpressed EBP50 with GFP-wtCFTR, GFP-CFTR- $\triangle$ TRL, or GFP-CFTR-S1455X in COS cells and determined whether CFTR and EBP50 could be coimmunoprecipitated. EBP50 coimmunoprecipitated with GFP-wt-CFTR but not with GFP-CFTR- $\Delta$ TRL (Figure 4) or GFP-S1455X. These observations demonstrate that CFTR-EBP50 coimmunoprecipitation requires the COOH-terminal PDZ-interacting domain of CFTR. Taken together, these observations suggest that the polarized expression of CFTR at the apical membrane requires the $\mathrm{PDZ}$-interacting domain of CFTR and may involve interaction with EBP50.

\section{Discussion}

We have demonstrated that the last 3 amino acids in the COOH-terminus of CFTR (T-R-L) comprise a PDZ-interacting domain that is required for the polarization of CFTR to the apical plasma membrane in human airway and kidney epithelial cells. Our data also suggest that the polarization of CFTR to the apical plasma membrane involves interaction with an apical membrane PDZ
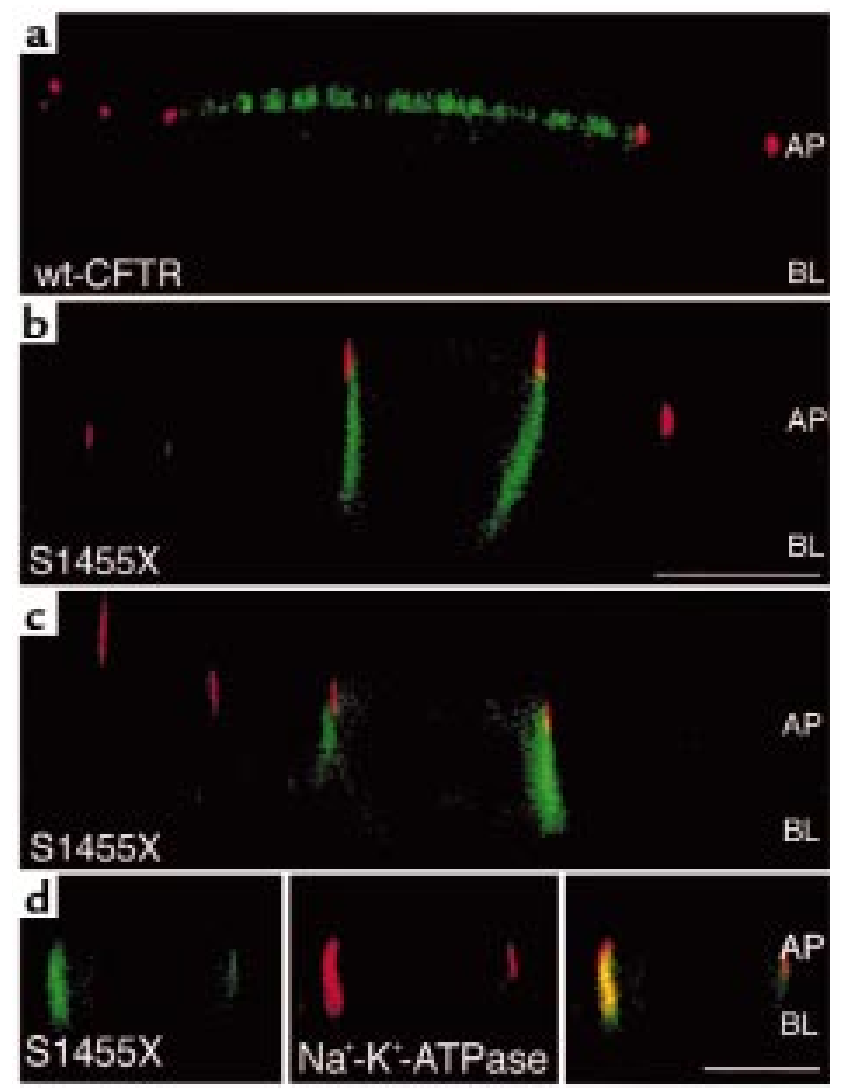

\section{Figure 2}

Confocal fluorescence micrographs (xz plane) of cells expressing GFP-wtCFTR or GFP-CFTR-S1455X. GFP fluorescence is green, and ZO-1, a protein in tight junctions that separates apical and basolateral membrane domains, is red. (a) GFP-wt-CFTR is located in the apical membrane of MDCK cells. (b) GFP-CFTR-S1455X is located in the lateral membrane of MDCK cells. (c) GFP-CFTR-S1455X is located in the lateral membrane of 16HBE14o- cells. (d) GFP-CFTR-S1455X (left panel in green) colocalizes with $\mathrm{Na}^{+}-\mathrm{K}^{+}$-ATPase (middle panel in red) in the lateral membrane (right panel is a merge of red and green channels, yellow-orange indicates colocalization). GFP-wt-CFTR is expressed in the apical membrane of 16HBE14o- cells (image not shown). Note that some GFP-CFTR-S1455X is expressed in an intracellular compartment. Scale bars $=10 \mu \mathrm{m} . \mathrm{AP}=$ location of apical membrane; $\mathrm{BL}=$ location of basal membrane. 

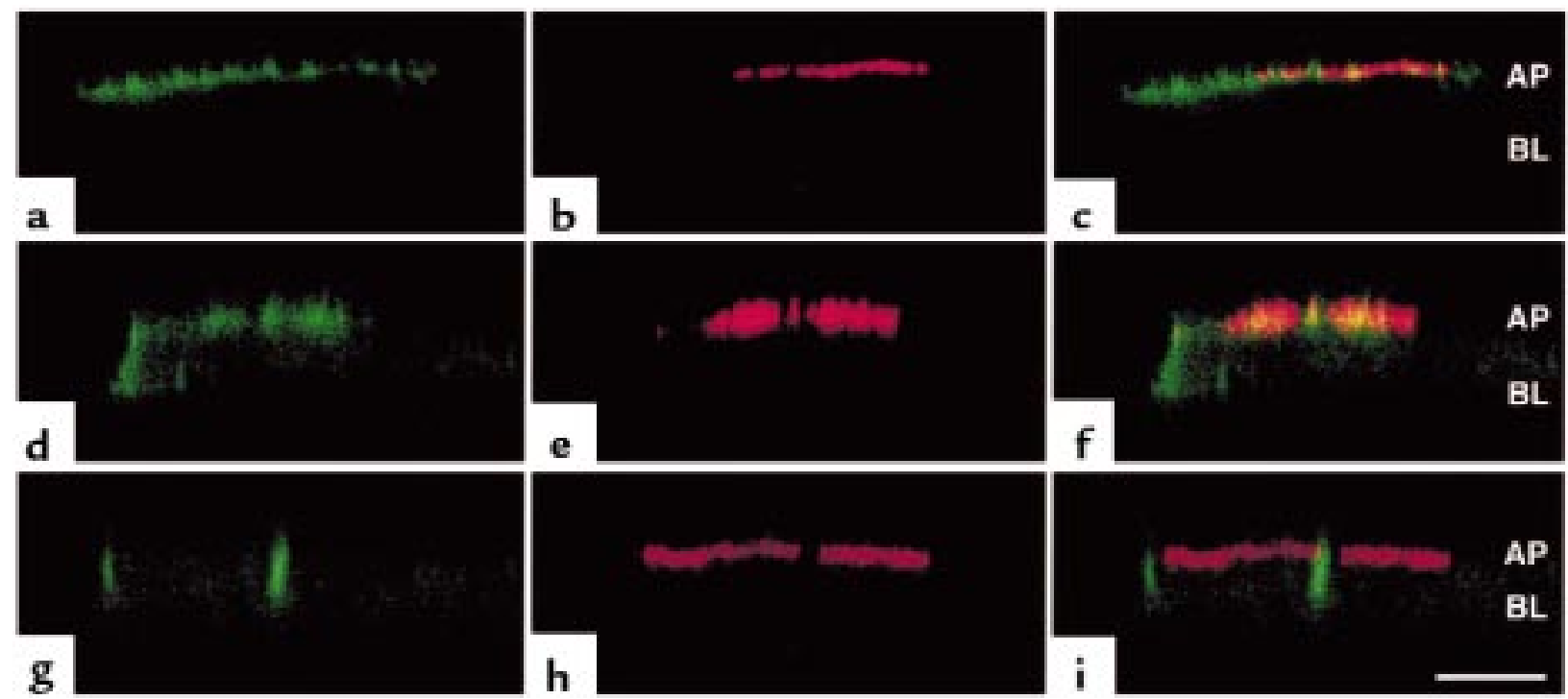

\section{Figure 3}

Deletion of the PDZ-interacting domain of CFTR abrogates colocalization of CFTR and EBP50. Confocal fluorescence micrographs (xz plane) of MDCK cells coexpressing: (a-c) EBP50 and wt-CFTR, (d-e) EBP50 and CFTR- $\triangle T R L$, and (g-i) EBP50 and CFTR-S1455X. GFP-CFTR is green (a, d, $\mathbf{g}$ ), EBP50 is red ( $\mathbf{b}, \mathbf{e}, \mathbf{h})$, and the merged red and green images are shown in $\mathbf{c}, \mathbf{f}$, and $\mathbf{i}$. Colocalization of EBP50 and GFP-CFTR is orange-yellow in $\mathbf{c}$ and $\mathbf{f}$. Note that some GFP-CFTR-S1455X and GFP-CFTR- $\Delta$ TRL are expressed in an intracellular compartment ( $\mathbf{d}$ and $\mathbf{g}$ ). Also note that only some cells express EBP50 because EBP50 is transiently expressed in approximately $10 \%$ of cells. Scale bar $=10 \mu \mathrm{m}$. AP $=$ location of apical membrane; BL $=$ location of basal membrane.

domain-containing protein such as EBP50, although we cannot exclude the possibility that CFTR may interact with PDZ domains in other proteins such as CFTR-associated ligand (CAL) $(41,42)$. Thus, we propose that $\mathrm{COOH}$-terminal deletions of CFTR, which represent about $10 \%$ of CFTR mutations and cause severe CF phenotypes, may result in defective vectorial chloride transport in part by altering the polarized distribution of CFTR in epithelial cells. Moreover, our data support the emerging concept that interaction between PDZ domains and PDZ-interacting domains plays a key role in the apical polarization of ion channels in epithelial cells.

Interaction between PDZ domains and the PDZ-interacting domain located in the $\mathrm{COOH}$-terminus of many proteins has been shown to be required for their polarized expression in epithelial cells. For example, in Caenorbabditis elegans, the receptor tyrosine kinase let-23 contains a PDZ-interacting domain and is localized to the lateral membrane by direct interaction with the PDZ domain-containing lin-7/lin-2/lin-10 complex $(24,27)$. Mutations in the PDZ domain of lin-7 causes let-23 to redistribute from the lateral to the apical membrane (24, 27). Lin-10 is also required for basolateral localization of GLR-1 glutamate receptors in C. elegans (26). In mammalian cells, the proteoglycan syndecan contains a PDZinteracting domain and is localized to the lateral membrane by CASK, the mammalian homologue of lin-2 (43). Deletion of the syndecan $\mathrm{COOH}$-terminus results in nonpolarized expression to apical and basolateral membranes (44). Thus, interactions between PDZ-interacting domains and lin-7/lin-2/lin-10 homologs may be a general mechanism for basolateral polarization in epithelial cells. A role for PDZ domains in determining the polarization of proteins to the apical membrane is also emerg- ing. For example, a splice variant of the voltage-gated $\mathrm{K}^{+}$ channel $\mathrm{K}_{\mathrm{v} 3.2 \mathrm{a}}$, which contains a $\mathrm{COOH}$-terminal PDZinteracting domain-like sequence, is polarized to the apical membrane (29). By contrast, a splice variant that deletes the $\mathrm{COOH}$-terminal PDZ-interacting domain-like sequence of $\mathrm{K}_{\mathrm{v} 3.2 \mathrm{a}}$ is polarized to the basolateral membrane (29). In addition, the neuronal GABA-3 transporter (GAT-3) contains a COOH-terminal, PDZ-interacting domain-like sequence and is polarized to the apical membrane (28). Deletion of the $3 \mathrm{COOH}$-terminal amino acids disrupts the apical polarization of GAT-3 and results in nonpolarized expression in apical and basolateral domains (28). Thus, our results are consistent with the emerging view that interaction between PDZ domains and PDZ-interacting domains is involved in the polarized expression of ion channels and other transport proteins in the apical plasma membrane of epithelia.

Our results are consistent with the view that EBP50, or another PDZ domain-containing protein such as CAL $(41,42)$, may play a key role in the polarization of CFTR to the apical plasma membrane in epithelial cells. Deletion of the PDZ-interacting domain of CFTR abrogated colocalization of CFTR and EBP50 to the apical membrane and eliminated coimmunoprecipitation of EBP50 and CFTR. Interaction of CFTR with EBP50 may mediate the polarized expression of CFTR to the apical plasma membrane by several mechanisms. First, upon sorting to the apical plasma membrane, CFTR may be retained or anchored by binding to PDZ domains. Similar mechanisms mediate the polarized expression of $\mathrm{Na}^{+}-\mathrm{K}^{+}$-ATPase and $\beta 1$-integrin in the basolateral membrane, where interaction with the actin-based cytoskeleton retains these proteins in the basolateral membrane (17-19). In some cell lines, $\mathrm{Na}^{+}-\mathrm{K}^{+}$-ATPase is sorted from 
the trans-Golgi network to both the apical and basolateral membrane: however, the $\mathrm{Na}^{+}-\mathrm{K}^{+}$-ATPase that is sorted to the apical membrane is rapidly endocytosed and degraded, whereas the $\mathrm{Na}^{+}-\mathrm{K}^{+}$-ATPase sorted to the basolateral membrane is retained and accumulates in this membrane via its interaction with the actin-based cytoskeleton, thereby leading to basolateral polarization (17). Second, EBP50, or another PDZ domain-containing protein such as $\mathrm{CAL}$, may facilitate anterograde trafficking of CFTR along the secretory pathway from the endoplasmic reticulum to the Golgi to the apical plasma membrane. This view is supported by the recent observation that CFTR associates with CAL in the trans-Golgi network (42) and that the PDZ-interacting domain of pro-TGF- $\alpha$ is required for its efficient trafficking along the secretory pathway and for its expression at the plasma membrane (45). Moreover, the COOH-most terminal amino acids of several proteins are required for the efficient export of the proteins from the endoplasmic reticulum and/or the trafficking of these proteins to the plasma membrane $(46,47)$. Third, the PDZ-interacting domain may be a apical sorting determinant that directs the trafficking of CFTR from the trans-Golgi network to the apical membrane. In the present study, we observed that approximately $30 \%$ of total cellular GFP-wt-CFTR was expressed in the plasma membrane, whereas only $1-3 \%$ of total cellular GFP-CFTR- $\Delta$ TRL and GFP$\mathrm{S} 1455 \mathrm{X}$ was expressed in the plasma membrane. These observations are consistent with all 3 hypothesis presented here. Interestingly, elimination of EBP50-CFTR interaction abrogates the apical polarization of CFTR but does not alter the apical localization of EBP50 (Figure 3). Thus, it is possible that PDZ domain-containing proteins stabilize CFTR in the apical membrane and/or direct the trafficking of CFTR to the apical membrane. However, elucidation of the mechanism whereby the PDZ-interacting domain of CFTR directs its apical polarization requires additional study.

EBP50, like other PDZ domain-containing proteins, may organize 3 -dimensional macromolecular signaling complexes at the apical plasma membrane of epithelial cells, thereby linking CFTR with regulatory kinases such as protein kinase A, ezrin (a protein kinase A anchoring protein that binds to actin [48]), phosphatases, and other apical membrane ion channels, such as outwardly rectifying chloride channels (ORCC), the epithelial sodium channel $(\mathrm{ENaC})$, and the epithelial $\mathrm{K}^{+}$channel (ROMK), which functionally interact with CFTR (49). According to this view, disruption of PDZ domain-CFTR interaction would result in defective regulation of CFTR by kinases and phosphatases and abrogation of CFTR regulation of ENaC, ORCC, and ROMK. Additional studies are required to determine whether CFTR-EBP50 interaction is required for CFTR to regulate the function of other proteins and to be regulated by kinases and phosphatases.

Although the polarization of CFTR-S1455X is dramatically different from wt-CFTR, this mutation does not cause $\mathrm{CF}$, as pulmonary and pancreatic function appear to be normal; however, CFTR-S1455X results in elevated sweat chloride concentration (40). Because CFTR-S1455X is a functionally normal $\mathrm{Cl}^{-}$channel, we conclude that defective $\mathrm{Cl}^{-}$transport in sweat ducts is most likely due to the mispolarization of CFTR-S1455X to the lateral rather than the apical plasma membrane. We speculate that the relatively small fraction of CFTR-S1455X expressed at the apical membrane may be sufficient to sustain pulmonary and pancreatic function, but not sweat duct function. In addition, the fact that CFTR-S1455X was polarized to the lateral membrane, whereas CFTR- $\Delta$ TRL was equally distributed between the apical and basolateral plasma membranes, suggests that some basolateral sorting information is suppressed in CFTR- $\Delta$ TRL. Deletion of the last 26 amino acids of CFTR may unmask suppressed basolateral sorting motifs such as the tyrosine-based motif (amino acids 1424-1427) or the dileucine motif (amino acids 1430-1431). Because sorting determinants are position dependent and hierarchical, deletion of the last 26 amino acids in CFTR may unmask the tyrosine and/or dileucine motifs, such that 1 or both of these motifs direct CFTRS1455X to the lateral membrane $(13,14)$.

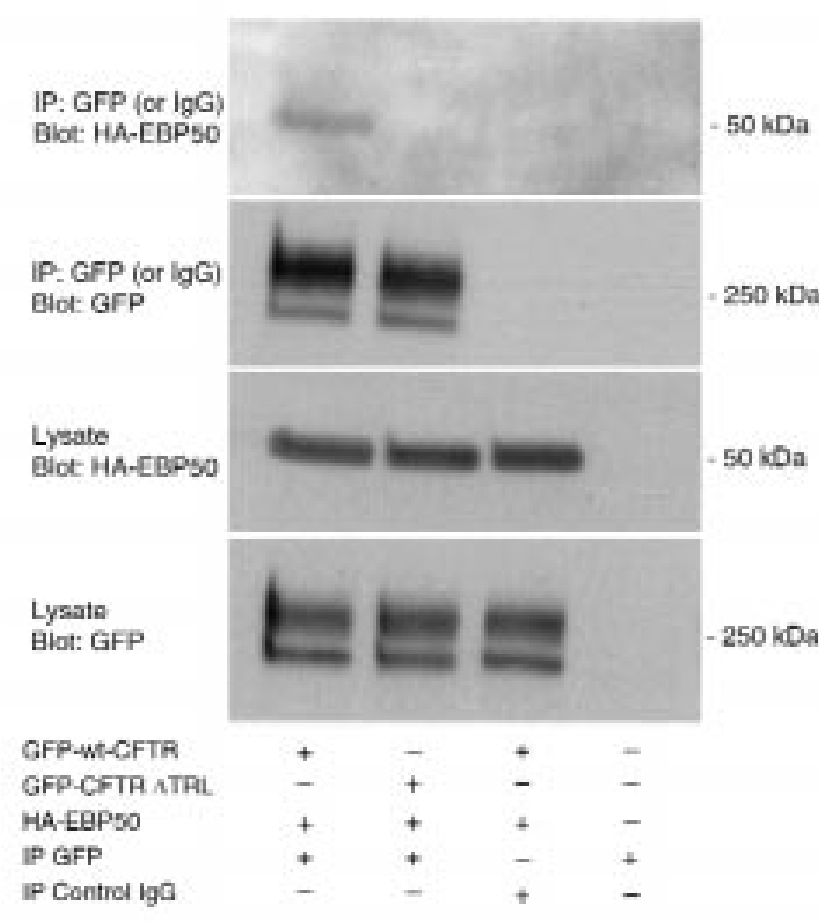

\section{Figure 4}

Coimmunoprecipitation of EBP50 and CFTR. EBP50 was coexpressed with GFP-wt-CFTR or GFP-CFTR- $\Delta$ TRL in COS-7 cells. Immunoprecipitation was conducted with a polyclonal GFP antibody (to immunoprecipitate CFTR) or a nonspecific, control IgG antibody and blots were probed with monoclonal GFP (to detect CFTR) or an anti-HA antibody to detect HA-tagged EBP50, as indicated. Cell lysates were probed for the expression of CFTR and EBP50. Top 2 blots show immunoprecipitations. Bottom 2 blots show cell lysates. Neither EBP50 nor CFTR was immunoprecipitated when a nonspecific, control $\lg G$ from nonimmune rabbit serum was used. CFTR immunoprecipitation efficiency was similar in all samples, and cell lysates expressed equivalent levels of EBP50 and CFTR fusion proteins. Thus, results cannot be attributed to differential protein expression. Similar results were obtained when EBP50 was immunoprecipitated and the blots probed with a GFP antibody to identify CFTR (data not shown). Mature glycosylated GFP-wt-CFTR band C is approximately $240 \mathrm{kDa}$. The higher molecular weight band of CFTR has been reported $(33,36)$. 
Several classes of mutations in CFTR have been identified, including those that affect protein synthesis (class I); protein folding, processing, and export from the endoplasmic reticulum to the plasma membrane (class II); regulation by ATP and protein kinase A (class III); ion conductance (class IV); stability or abundance of mRNA and protein (class $\mathrm{V}$ ); and regulation of other ion channels (class VI) (5). Because deletion of the COOH-terminus of CFTR (e.g., up to 61 amino acids) does not affect the ability of cAMP to activate CFTR-mediated $\mathrm{Cl}^{-}$currents $(38,39)$, yet causes severe CF, we speculate that mutations that delete the COOH-terminus of CFTR, including the PDZ-interacting domain, may abrogate vectorial $\mathrm{Cl}^{-}$transport in part by disrupting CFTR polarization to the apical membrane. Indeed, in MDCK cells stably expressing GFP-CFTR- $\triangle$ TRL or GFP-CFTRS1455X, we observed that CPT-cAMP-stimulated, transepithelial $\mathrm{Cl}^{-}$secretion was not different from parental, untransfected MDCK cells. By contrast, as reported previously, MDCK cells stably expressing GFPwt-CFTR manifest significantly higher rates of CPTcAMP-stimulated transepithelial $\mathrm{Cl}^{-}$secretion compared with parental untransfected MDCK cells (33). Thus, mutations that delete the $\mathrm{COOH}$-terminus may constitute a new class of CFTR mutations (class VII) that cause defective $\mathrm{Cl}^{-}$secretion because $\mathrm{COOH}$-terminal truncated CFTR is mispolarized to the lateral membrane. Additional studies on other $\mathrm{COOH}$-terminal deletions of CFTR are required to support this hypothesis.

Our observation that $\mathrm{COOH}$-terminal truncations abrogate CFTR polarization in epithelial cells may have important implications for CFTR gene therapy. Recently, it was proposed that $\mathrm{COOH}$-terminal truncations of CFTR, which increase transfection efficiency, may be useful for human gene therapy (39). However, because $\mathrm{COOH}$-terminal deletions of CFTR affect its polarized distribution in epithelial cells, it must be considered that these deletion constructs may not lead to improved apical membrane expression compared with wt-CFTR in polarized epithelial cells in vivo.

In summary, we report that the last 3 amino acids in the COOH-terminus of CFTR (T-R-L) comprise a PDZinteracting domain that is required for the polarization of CFTR to the apical plasma membrane in human airway and kidney epithelial cells. Our data also suggest that apical polarization of CFTR involves interaction with EBP50, or some other PDZ domain-containing protein such as CAL. We propose that $\mathrm{COOH}$-terminal deletions of CFTR, which represent about $10 \%$ of CFTR mutations, may result in defective vectorial chloride transport in part by altering the polarized distribution of CFTR in epithelial cells. Moreover, our data demonstrate that PDZ-interacting domains and PDZ domain-containing proteins play a key role in the apical polarization of ion channels in epithelial cells.

\section{Acknowledgments}

We thank Alice Givan, Ken Orndorff, and Gary Ward for assistance with confocal microscopy and flow cytometry; Olivia Weale for assistance with plasmid preparations; and Barack Abonyo for work done during the early phase of this study. This work was supported by the Cystic Fibro- sis Foundation and by grants from the National Institutes of Health (HL-47122, DK-48977, and DK-32753 to W.B. Guggino; RO1-NS33334 to M. Li; and DK-45881 and DK51067 to B.A. Stanton). B.D. Moyer was supported by a predoctoral grant from the Dolores Zohrab Liebmann Foundation. S. Wang was supported by a postdoctoral fellowship from the Cystic Fibrosis Foundation.

1. Crawford, I., et al. 1991. Immunocytochemical localization of the cystic fibrosis gene product CFTR. Proc. Natl. Acad. Sci. USA. 88:9262-9266.

2. Denning, G.M., Ostedgaard, L.S., Cheng, S.H., Smith, A.E., and Welsh, M.J. 1992. Localization of cystic fibrosis transmembrane conductance regulator in chloride secretory epithelia. J. Clin. Invest. 89:339-349.

3. Stanton, B.A. 1997. Cystic fibrosis transmembrane conductance regulator (CFTR) and renal function. Wien. Klin. Wochenschr. 109:457-464.

4. Davis, P.B., Drumm, M., and Konstan, M.W. 1996. Cystic fibrosis. Am.J. Respir. Crit. Care Med. 154:1229-1256.

5. Mickle, J.E., and Cutting, R.G. 1998. Clinical implications of cystic fibrosis transmembrane conductance regulator mutations. Clin. Chest Med. 19:443-458.

6. Riordan, J.R., et al. 1989. Identification of the cystic fibrosis gene: cloning and characterization of complementary DNA. Science. 245:1066-1073.

7. Cystic Fibrosis Mutation Data Base. http://www.genet.sickkids.on.ca/cftr. 8. Cheng, S.H., et al. 1990. Defective intracellular transport and processing of CFTR is the molecular basis of most cystic fibrosis. Cell. 63:827-834.

9. Jilling, T., and Kirk, K.L. 1997. The biogenesis, traffic, and function of the cystic fibrosis transmembrane conductance regulator. Int. Rev. Cytol. 172:193-241.

10. Pasyk, E.A., and Foskett, J.K. 1997. Cystic fibrosis transmembrane conductance regulator-associated ATP and adenosine 3 '-phosphate $5^{\prime}$-phosphosulfate channels in endoplasmic reticulum and plasma membranes. J. Biol. Chem. 272:7746-7751.

11. Aroeti, B., Okhrimenko, H., Reich, V., and Orzech, E. 1998. Polarized trafficking of plasma membrane proteins: emerging roles for coats, SNAREs, GTPases and their link to the cytoskeleton. Biochim. Biophys. Acta Rev. Biomembr. 1376:57-90.

12. Bradke, F., and Dotti, C.G. 1998. Membrane traffic in polarized neurons. Biochim. Biophys. Acta Mol. Cell Res. 1404:245-258.

13. Brown, D., and Stow, J.L. 1996. Protein trafficking and polarity in kidney epithelium: from cell biology to physiology. Physiol. Rev. 76:245-297.

14. Caplan, M.J. 1997. Ion pumps in epithelial cells: sorting, stabilization, and polarity. Am. J. Physiol. Gastrointest. Liver Physiol. 272:G1304-G1313.

15. Matter, K., and Mellman, I. 1994. Mechanisms of cell polarity: sorting and transport in epithelial cells. Curr. Opin. Cell Biol. 6:545-554.

16. Mays, R.W., Nelson, W.J., and Marrs, J.A. 1995. Generation of epithelial cell polarity: roles for protein trafficking, membrane-cytoskeleton, and E-cadherin-mediated cell adhesion. Cold Spring Harbor Symp. Quant. Biol. LX:763-773.

17. Hammerton, R.W., et al. 1991. Mechanism for regulating cell surface distribution of $\mathrm{Na}^{+}, \mathrm{K}^{+}$-ATPase in polarized epithelial cells. Science. 254:847-850.

18. Morrow, J.S., Cianci, C.D., Ardito, T., Mann, A.S., and Kashgarian, M. 1989. Ankyrin links fodrin to the alpha subunit of Na,K-ATPase in Madin-Darby canine kidney cells and in intact renal tubule cells. J. Cell Biol. 108:455-465

19. Gut, A., Balda, M.S., and Matter, K. 1998. The cytoplasmic domains of a $\beta_{1}$ integrin mediate polarization in Madin-Darby canine kidney cells by selective basolateral stabilization. J. Biol. Chem. 273:29381-29388.

20. Fanning, A.S., and Anderson, J.M. 1999. PDZ domains: fundamental building blocks in the organization of protein complexes at the plasma membrane. J. Clin. Invest. 103:767-772.

21. Kornau, H.C., Seeburg, P.H., and Kennedy, M.B. 1997. Interaction of ion channels and receptors with PDZ domain proteins. Curr. Opin. Neurobiol. 7:368-373.

22. Saras, J., and Heldin, C.H. 1996. PDZ domains bind carboxy-terminal sequences of target proteins. Trends Biochem. Sci. 21:455-458.

23. Tsunoda, S., et al. 1997. A multivalent PDZ-domain protein assembles signalling complexes in a G-protein-coupled cascade. Nature. 388:243-249.

24. Kaech, S.M., Whitfield, C.W., and Kim, S.K. 1998. The LIN-2/LIN-7/LIN10 complex mediates basolateral membrane localization of the C. elegans EGF receptor LET-23 in vulval epithelial cells. Cell. 94:761-771.

25. Perego, C., et al. 1999. PDZ-mediated interactions retain the epithelial GABA transporter on the basolateral surface of polarized epithelial cells. EMBO J. 18:2384-2393.

26. Rongo, C., Whitfield, C.W., Rodal, A., Kim, S.K., and Kaplan, J.M. 1998. LIN-10 is a shared component of the polarized protein localization pathways in neurons and epithelia. Cell. 94:751-759.

27. Simske, J.S., Kaech, S.M., Harp, S.A., and Kim, S.K. 1996. LET-23 recep- 
tor localization by the cell junction protein LIN-7 during C. elegans vulval induction. Cell. 85:195-204.

28. Muth, T.R., Ahn, J., and Caplan, M.J. 1998. Identification of sorting determinants in the C-terminal cytoplasmic tails of the gammaaminobutyric acid transporters GAT-2 and GAT-3. J. Biol. Chem. 273:25616-25627.

29. Ponce, A., et al. 1997. K+ channel subunit isoforms with divergent carboxy-terminal sequences carry distinct membrane targeting signals. $J$. Membr. Biol. 159:149-159.

30. Hall, R.A., et al. 1998. A C-terminal motif found in the $\beta 2$-adrenergic receptor, $\mathrm{P} 2 \mathrm{Y} 1$ receptor and cystic fibrosis transmembrane conductance regulator determines binding to the $\mathrm{Na}^{+} / \mathrm{H}+$ exchanger regulatory factor family of PDZ proteins. Proc. Natl. Acad. Sci. USA. 95:8496-8501.

31. Short, D.B., et al. 1998. An apical PDZ protein anchors the cystic fibrosis transmembrane conductance regulator to the cytoskeleton. J. Biol. Chem. 273:19797-19801.

32. Wang, S.S., Raab, R.W., Schatz, P.J., Guggino, W.B., and Li, M. 1998. Peptide binding consensus of the NHE-RF-PDZ1 domain matches the Cterminal sequence of cystic fibrosis transmembrane conductance regulator (CFTR). FEBS Lett. 427:103-108.

33. Moyer, B.D., et al. 1998. Membrane trafficking of the cystic fibrosis gene product, cystic fibrosis transmembrane conductance regulator, tagged with green fluorescent protein in Madin-Darby canine kidney cells. $J$. Biol. Chem. 273:21759-21768.

34. Johnston, J.A., Ward, C.L., and Kopito, R.R. 1998. Aggresomes: a cellular response to misfolded proteins. J. Cell Biol. 143:1883-1898.

35. Gregory, R.J., et al. 1990. Expression and characterization of the cystic fibrosis transmembrane conductance regulator. Nature. 347:382-386.

36. Marshall, J., et al. 1994. Stoichiometry of recombinant cystic fibrosis transmembrane conductance regulator in epithelial cells and its functional reconstitution into cells in vitro. J. Biol. Chem. 269:2987-2995.

37. Gottardi, C.J., and Caplan, M.J. 1993. An ion-transporting ATPase encodes multiple apical localization signals. J. Cell Biol. 121:283-293.
38. Rich, D.P., Gregory, R.J., Cheng, S.H., Smith, A.E., and Welsh, M.J. 1993. Effect of deletion mutations on the function of CFTR chloride channels. Receptors Channels. 1:221-232.

39. Zhang, L., et al. 1998. Efficient expression of CFTR function with adenoassociated virus vectors that carry shortened CFTR genes. Proc. Natl. Acad. Sci. USA. 95:10158-10163.

40. Mickle, J.E., et al. 1998. A mutation in the cystic fibrosis transmembrane conductance regulator gene associated with elevated sweat chloride concentrations in the absence of cystic fibrosis. Hum. Mol. Genet. 7:729-735.

41. Cheng, J., and Guggino, W.B. 1998. Molecular cloning and characterization of a novel PDZ domain containing protein that interacts with CFTR. Pediatr. Pulmonol. 17(Suppl.):213. (Abstr.)

42. Cheng, J., et al. 1999. CFTR associates with the PDZ domain protein CAL at the trans-Golgi network. Pediatr. Pulmonol. Suppl. 19:168. (Abstr.)

43. Cohen, A.R., et al. 1998. Human CASK/LIN-2 binds syndecan-2 and protein 4.1 and localizes to the basolateral membrane of epithelial cells. J. Cell. Biol. 142:129-138.

44. Miettinen, H.M., Edwards, S.N., and Jalkanen, M. 1994. Analysis of transport and targeting of syndecan-1: effect of cytoplasmic tail deletions. Mol. Biol. Cell. 5:1325-1339.

45. Fernandez-Larrea, J., Merlos-Suarez, A., Urena, J.M., Baselga, J., and Arribas, J. 1999. A role for a PDZ protein in the early secretory pathway for the targeting of proTGF- $\alpha$ to the cell surface. Mol. Cell. 3:423-433.

46. Ciruela, F., Soloviev, M.M., and McIlhinney, R.A. 1999. Cell surface expression of the metabotropic glutamate receptor type 1alpha is regulated by the C-terminal tail. FEBS Lett. 448:91-94.

47. Nakamura, N., et al. 1998. Identification of potential regulatory elements for the transport of Emp24p. Mol. Biol. Cell. 9:3493-3503.

48. Dransfield, D.T., et al. 1997. Ezrin is a cyclic AMP-dependent protein kinase anchoring protein. EMBOJ. 16:35-43.

49. Kunzelmann, K. 1999. The cystic fibrosis transmembrane conductance regulator and its function in epithelial transport. Rev. Physiol. Biochem. Pharmacol. 137:1-70. 